\title{
An Efficient Reduced Order Modeling Technique for Nonlinear Vibration Analysis of Structures with Intermittent Contact
}

\author{
Akira Saito* and Matthew P. Castanier ${ }^{\dagger}$ \\ Department of Mechanical Engineering \\ The University of Michigan, Ann Arbor, MI 48109-2125, USA \\ Christophe Pierre $\ddagger$ \\ Faculty of Engineering \\ McGill University, Montreal, Quebec, H3A 2K6, Canada
}

\begin{abstract}
In this paper, a reduced order modeling framework for nonlinear vibration problems of elastic structures involving intermittent contact is proposed. Of particular interest is a vibration problem of plate-like elastic structures with a crack with a large number of degrees of freedom involved on the crack surfaces. Due to the localized nature of such nonlinearity, the number of degrees of freedom on the surfaces greatly affects the computational time of the analysis. Therefore, reducing the number of degrees of freedom on the crack surfaces without significantly sacrificing the accuracy of the results is a critical issue for conducting vibration analysis of such structures in a reasonable amount of time. The focus is placed on the development of an efficient algorithm to select a set of nodes on the crack surfaces, where nonlinear boundary conditions are imposed. The method is developed based on a procedure for selecting master degrees of freedom for Guyan reduction. The accuracy, efficiency, and optimality of the method are discussed in detail and compared with those aspects of previous methods. The advantages of the new method are demonstrated in terms of the accuracy of the frequency response and the resonant frequencies.
\end{abstract}

\section{Introduction}

$\mathrm{V}$ IBRATION problems of structures with intermittent contact have been studied extensively for several decades. These problems have practical importance and feature theoretical complexity due to their nonlinear nature. A numerical modeling procedure of such problems based on the finite element (FE) method is presented in this paper. This work is motivated by a need for developing a model-based crack detection algorithm of elastic structures based on their spectral properties, such as resonant frequencies and response shapes. In order to properly predict the resonant frequencies of such structures, one has to consider the nonlinearity caused by intermittent contact at the cracks, the so-called closing crack or breathing crack effect. This has hindered analysts from accurately calculating the resonant frequencies of cracked structures, because they cannot be calculated from classical linear modal analysis. As some sophisticated contact algorithms have been developed, such as the penalty $\operatorname{method}^{1}$ and the augmented Lagrangian method, ${ }^{2}$ the accuracy of the results of time transient simulation with FE models involving intermittent contact has been improved. Furthermore, studying vibration problems of such structures with an FE model with a realistic complexity is becoming feasible with the aid of high-performance computers. However, in turn, due to

\footnotetext{
${ }^{*}$ Graduate Student Research Assistant, Student Member of AIAA

${ }^{\dagger}$ Associate Research Scientist, Senior Member of AIAA

${ }^{\ddagger}$ Dean, Senior Member of AIAA
} 
the advancement of these technologies, analysts tend to create models with a large number of degrees of freedom (DOF). This is based on the expectation that, as the model becomes more realistic and the results become more accurate, the problem can still be solved in a reasonable amount of time. However, at some point the number of DOF will overwhelm even the most advanced hardware and software. In fact, as the model complexity increases, the cost of solving contact problems increases dramatically, even when the potential contact areas are known a priori. This occurs even if one uses reduced order modeling techniques, such as the Craig-Bampton method. ${ }^{3}$ For forced response vibration problems of such structures, one can use accurate and efficient semi-analytical methods such as the ones based on the harmonic balance method (e.g., Ref. ${ }^{4}$ ), by representing the steady-state dynamic response of the model with a truncated Fourier series. However, such methods still suffer from the increase of computational cost as it requires a fair number of harmonics to be included for the Fourier transform, in order to obtain an accurate result. Therefore, the goal of this paper is to propose an efficient reduced order modeling framework for vibration problems of elastic structures involving intermittent contact, with particular attention to modeling nonlinear vibration of cracked structures. The focus is placed upon reducing the number of DOF involved in the contact regions, in an automatic manner.

This paper is organized as follows. In section II, a literature survey over the related fields is provided. In section III, the proposed modeling framework is presented, including the reduced order modeling approach and contact DOF selection method. As applications of the method, a case study is shown in section IV, using an FE model of a cantilevered cracked plate. Conclusions of the paper are then given in section V.

\section{Background}

The issues of reducing and selecting DOF of FE models have been extensively studied by various methods and perspectives, such as the reduction of the interface DOF between substructures, selection of master DOF for Guyan reduction, ${ }^{5}$ optimal sensor placement, and optimal constraint locations. However, many of the available methods share to some extent similar goals and related to each other as described later.

Firstly, the issue of reducing the number of interface DOF between the components has been studied by several researchers. Brahmi et al. ${ }^{6}$ proposed a method for reducing the number of interface DOF before the assembly of substructures, where basis vectors are chosen based on the combination of secondary modal analysis of the interface DOF partitions of the matrices, and the truncation of modes based on the singular value decomposition. Balmés ${ }^{7}$ introduced the framework for generalizing interface DOF such as constraint modes, by considering the new basis representing the actual interface displacements. Castanier et al. ${ }^{8}$ also proposed a technique for reducing the number of interface DOF by applying the modal analysis and mode truncation to the constraint mode partition of the matrices produced by the Component Mode Synthesis (CMS), ${ }^{3}$ the resulting modes of which are called the characteristic constraint modes after being transformed back into the finite element coordinates. All of these methods achieve the order reduction of the DOF at the interface. However, they do not provide any criteria as to how the interface DOF need be selected for accurately enforcing the boundary conditions.

Secondly, the selection of master DOF has been a crucial factor for determining the spectral property of the reduced order model for Guyan reduction-based reduction techniques, and many algorithms for the selection of the master DOF have been developed. As it shall be discussed later, this class of methods produces the results that tend to solve the optimization problem for the problems studied in this study, thus it is very relevant to our objective. An automatic master DOF selection algorithm was first proposed by Henshell and Ong, ${ }^{9}$ in which the master DOF are chosen where the inertia is high and the stiffness is low, whereas the slave DOF are chosen where the inertia is low and the stiffness is high. This process can be automated by examining the radian frequency $\omega_{s}$ defined by fixing all DOF except the DOF index $s$. Namely, $\omega_{s} \triangleq \sqrt{k_{s s} / m_{s s}}$, for $s=1, \ldots, n$, where $k_{i j}$ and $m_{i j}$ are the entries at the $i$ th row and $j$ th column in FE stiffness and mass matrices of size $n$. The index $s$ with the largest $\omega_{s}$ is identified at each iteration step, and the DOF is eliminated by an application of Guyan reduction with $s$ being the slave DOF and all the 
other DOF being the master DOF. This process can be repeated until the number of master DOF reaches the desired number. An approach similar to this algorithm was proposed by Shah and Raymund ${ }^{10}$ based on the discussion of Kidder and Flax, ${ }^{11-13}$ where the number of master DOF is controlled by iteratively eliminating the DOF whose $\omega_{s}$ is larger than the pre-defined cut-off frequency $\omega_{c}$ that is chosen to be approximately three times the highest significant frequency in the frequency range of interest. Independently from the work by Henshell and Ong, Grinenko and Mokeev developed an order reduction technique named frequencydynamic condensation, ${ }^{14}$ which also proposed a criterion to select master DOF. Although their criterion was legitimate, the implementation of the selection algorithm still suffers from tedious exhaustive-search calculation for selecting the DOF. The selection method proposed by Matta ${ }^{15}$ also uses the ratio $k_{s s} / m_{s s}$ with the similar criterion to that proposed by Henshell and Ong. ${ }^{9}$ It was addressed that the method can be applied not only to the Guyan reduction but also to the CMS, where both static and vibration modes are used as basis vectors, onto which the system dynamics are projected. A method proposed by Bouhaddi and Fillod ${ }^{16}$ used a different concept where if a DOF $a$ is a node of an eigenmode, then fixing the DOF $a$ results in $\hat{\lambda}_{i}=\lambda_{i}$ where $\lambda_{i}$ is the $i$ th eigenvalue of the non-fixed system, and $\hat{\lambda}_{i}$ is the eigenvalue of the system with DOF $a$ being fixed. This concept may be understood using a vibration problem of a string with both ends fixed. That is, the lowest natural frequency of the string with a single support becomes the highest, if the support is placed at the node of the second mode of vibration. ${ }^{17}$ This is because the first mode with the constraint then becomes identical to the second mode of the unconstrained string, which has the eigenvalue as the feasible upper bound of the first eigenvalue with a single constraint. It is noteworthy that Bouhaddi and Fillod explicitly aimed for maximizing the minimum eigenvalue of the system where all the master DOF fixed. This concept will be revisited in III.C. The methods for the node selection reviewed so far are based on Henshell and Ong method to some extent. Another class of methods is that based on the concept of modal energy. The method proposed by Kim and Choi ${ }^{18}$ uses the energy distribution among the DOF for each mode, and by taking the partial sum over the rows of what they call energy distribution matrix, primary DOF set can be chosen. On the other hand the method proposed by Cho and Kim ${ }^{19}$ utilizes the energy estimation in element-level by the Rayleigh quotient value of each element. Kim and Cho then proposed a selection method consisting of two steps $;{ }^{20}$ model order reduction by Improved Reduced System (IRS) ${ }^{21}$ using the master DOF selected via a method based on energy estimation of each element, ${ }^{19}$ and subsequent sequential elimination method ${ }^{9}$ with an iterative IRS. Another automatic DOF selection method named modal energy selection method proposed by $\mathrm{Li}^{22}$ uses metric called index of classification, based on the approximate modal energy associated with each DOF. The method was successfully applied to an FE model of a cantilever beam problem. Oh and Park ${ }^{23}$ also proposed a criterion for selecting the master DOF based on singular values of the modal matrix, however, it suffers from the computational cost due to exhaustive search over the possible master DOF sets, and depends on engineer's knowledge and intuition.

Thirdly, a similar but slightly different issue is the selection of measurement locations for vibration testing. For example, one may need to measure vibration displacements of a structure to determine vibration modes, typically with a limited number of sensors or the locations where the sensors can be placed. Thus, one may like to maximize the information one can obtain as much as possible, with the limited number of sensors or locations. However the question arises as to how the sensors need to be located, since the optimal configuration of sensors for such objective cannot be easily determined. There have been many methods developed to date for achieving this goal with various approaches. In particular, one of the successful approaches are based on information theory, which determine the sensor locations by optimizing a norm of the Fisher information matrix. ${ }^{24}$ Among them, one of the most widely used techniques is the effective independence vector method, or the EIDV ${ }^{25}$ method developed by Kammer. ${ }^{26}$ The method determines the placement of sensors within the candidate locations while maintaining as much independent information as possible, i.e., maintaining the measured mode shapes as independent as possible. Therefore, it is natural to hypothesize that the application of the nonlinear boundary conditions to the optimum sensor locations would also well represent the real boundary conditions where the boundary conditions are applied to all locations in the region. This method is hereby considered in this study and the formulation is discussed in detail in 
III.C.

Lastly, the issue of finding the optimal constraint locations to maximize the fundamental natural frequency of a structure is considered. This issue has an important relationship with the optimal master DOF selection. For instance, suppose there is a structure that can vibrate, and one may want to increase the lowest natural frequency as much as possible, by allocating a finite number of supports or kinematical constraints to the structure. However, the problem of finding the optimal number and the locations of such supports is not as easy as it appears. Therefore, it may be necessary to apply mathematically expensive optimization algorithms to obtain such support locations, such as done in the work by Zhu and Zhang. ${ }^{27}$ On the other hand, Åkesson and Olhoff ${ }^{28}$ studied the problem by applying the Courant's maximum-minimum principle. Namely if there is a discrete dynamical system of size $n$ and there are $r(<n)$ kinematical constraints applied to the system, all the eigenvalues of the structure increase, and the increased eigenvalues are bounded by the following formula:

$$
\lambda_{i}^{0} \leqslant \lambda_{i} \leqslant \lambda_{i+r}^{0}, \quad i \in\{1,2, \ldots, n\}
$$

where $\lambda_{i}^{0}$ and $\lambda_{i+r}^{0}$ denote the $i$ th and $(i+r)$ th eigenvalues of the structure without the constraints, and $\lambda_{i}$ is the $i$ th eigenvalue of the constrained structure. Also based on the same principle and the findings of Ref., ${ }^{29}$ Won and Park ${ }^{30}$ applied minimization method to obtain the optimal support location to achieve the maximum fundamental natural frequency of a cantilevered plate. They showed that the optimal support locations should be on the nodal lines of the $(r+1)$ th mode of the unconstrained structure. It is noted that this result conforms to the vibration problem of a fixed string mentioned above. This method was successfully applied to their specific examples, but the method can be applied only to special cases if the potentially-constrained region is the entire region of the structure, where the points on the nodal lines can be selected. Namely, if the regions to which the constraints are applied are limited to some specific regions of the structure, then the nodal lines may not exist in such regions, and the minimization problem becomes more complicated.

It is interesting to note that the idea of constraining the nodal lines was used to optimally select the master DOF for Guyan reduction by Bouhaddi and Fillod, ${ }^{16}$ but they were not aware of the applicability of their method to optimally select the support positions, while Won and Park were not aware of the applicability of their method to optimally select the master DOF locations for Guyan reduction. In this paper, we take advantage of this similarity between the optimal master DOF selections and the constraint locations, in order to achieve the optimal selection of the DOF where the nonlinear boundary conditions are applied.

\section{Mathematical Formulation}

Consider small vibration problems of an elastic structure represented as $\Omega$ with a fixed boundary $\Gamma_{g}$, where the structure may or may not involve intermittent contact at $\Gamma_{A}$ and $\Gamma_{B}$ during the vibration cycles, such as shown in Fig. 1. Namely the boundaries open and close, thus the vibration problem is nonlinear because the condition for the the boundaries to be in contact is dependent on the displacement field itself. That is, the boundary conditions at $\Gamma_{A}$ and $\Gamma_{B}$ are nonlinear. It is well known that the system eigenvectors and eigen-frequencies are different from the actual response shapes and resonant frequencies of this nonlinear problem. In this paper, they are respectively referred to as the nonlinear normal modes (NNMs) and NNM frequencies.

Now, if the structure is discretized with a method such as finite element method, the nonlinearity associated with the contact is localized, in the sense that the nonlinearity is caused only by a small portion of the entire structure. In the following formulations, a set of indices of DOF in such region is denoted as $\mathcal{B}$ (boundary), whereas a set of indices of the DOF in the rest of the regions is denoted as $\mathcal{I}$ (internal), and partitions of vectors and matrices associated with these sets are designated with subscripts of the associated lower-case italic letters, i.e., $b$ and $i$. The sizes of the sets are denoted as $|\mathcal{B}|=n_{\mathcal{B}}$ and $|\mathcal{I}|=n_{\mathcal{I}}$. All the other DOF sets defined hereinafter follow the same notation. 


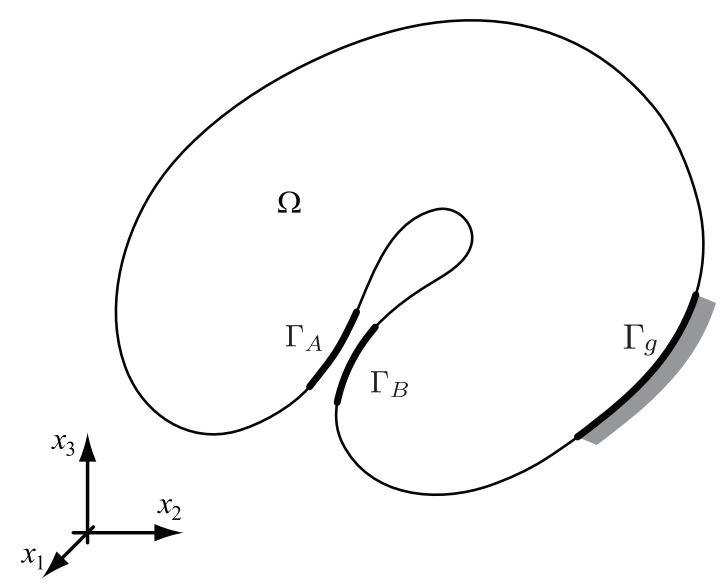

Figure 1. An elastic structure with potentially contacting boundaries

If the finite element mass and stiffness matrices are denoted as $\mathbf{M} \in \mathbb{R}^{n \times n}$ and $\mathbf{K} \in \mathbb{R}^{n \times n}$ and the nodal displacement vector is given as $\mathbf{x} \in \mathbb{R}^{n}$, the governing equations of the vibration problem with the absence of external forcing and damping may be written in a partitioned matrix-vector form as follows:

$$
\left(\begin{array}{ll}
\mathbf{M}_{b b} & \mathbf{M}_{b i} \\
\mathbf{M}_{i b} & \mathbf{M}_{i i}
\end{array}\right)\left[\begin{array}{l}
\ddot{\mathbf{x}}_{b} \\
\ddot{\mathbf{x}}_{i}
\end{array}\right]+\left(\begin{array}{ll}
\mathbf{K}_{b b} & \mathbf{K}_{b i} \\
\mathbf{K}_{i b} & \mathbf{K}_{i i}
\end{array}\right)\left[\begin{array}{l}
\mathbf{x}_{b} \\
\mathbf{x}_{i}
\end{array}\right]=\left[\begin{array}{c}
\mathbf{f}_{b}\left(\mathbf{x}_{b}\right) \\
\mathbf{0}
\end{array}\right]
$$

where a dot $\left({ }^{*}\right)$ denotes a time derivative, and $\mathbf{f}_{b} \in \mathbb{R}^{n_{\mathcal{B}}}$ denotes the nonlinear force associated with the intermittent contact. When dealing with this type of nonlinear vibration problems, one can apply linear reduced order modeling techniques, such as Guyan reduction, ${ }^{5}$ system equivalent reduction expansion process (SEREP), ${ }^{31}$ iterated improved reduced system (IIRS), ${ }^{32,33}$ or Component mode synthesis (CMS). ${ }^{3}$ With such methods, one can obtain smaller system matrices by reducing the size of $\mathbf{x}_{i}$ by means of Rayleigh-Ritz coordinate transformation comprising of various basis vectors such as static deformations and vibration modes, yet keeping the accessibility to the physical coordinates of $\mathbf{x}_{b}$. For instance, with the help of CMS, one can obtain a system with desired spectral properties and accessibility to $\mathbf{x}_{b}$, the size of which is as small as $n_{\mathcal{B}}$ DOF plus the number of linear normal modes whose frequencies lie in the frequency ranges of interest. The use of such linear reduced order modeling methods greatly helps ones to analyze the dynamic response of systems with localized nonlinearities, such as transient dynamic analysis, ${ }^{34}$ and nonlinear harmonic response analysis. ${ }^{35}$ However, even with these reduced order modeling methods, if the number of DOF involved in the $b$ partition becomes large, especially the cases with very fine mesh in the contacting regions, one cannot take advantage of the linear reduced order modeling techniques, as the computational cost associated with the nonlinear dynamic analysis typically grows as the number of DOF in the $b$ partition increases. Furthermore, if one simply attempts to eliminate some of the DOF in the $b$ partition, it results in inaccurate, or even wrong results, in comparison to the results obtained with a full set of DOF in the $b$ partition. Therefore, in order to obtain accurate computational results, such as those of nonlinear forced response, one needs to keep as many boundary DOF as possible, which could easily result in prohibitively costly calculations. Typically as a "workaround" to avoid the inaccurate results due to the lack of sufficient DOF considered and at the same time to obtain efficient computational model, one has to select the DOF in a heuristic way, which greatly depends on the system characteristics and analyst's experience and intuition. Moreover, if the model is developed in such ways, the error contained in the following analysis results cannot be estimated a priori. Our aim here is to develop an automatic way to select the DOF in $\mathcal{B}$ for a desired number of DOF to be selected. 


\section{III.A. Primary Model Reduction}

In order to reduce the number of DOF included in $\mathcal{I}$ to make the subsequent development more efficient, first a model reduction is applied to Eq. (2). Namely, $\mathcal{I}$ is further divided into two sets, i.e., $\mathcal{I}=\mathcal{O} \cup \mathcal{D}$ where $\mathcal{O}$ is a set of DOF indices associated with the nodes to be used in the following analysis, such as observing the behavior of the system or applying external loading, and $\mathcal{D}$ is the rest of DOF indices in $\mathcal{I}$, which is to be apparently deleted from the system by the reduction methods. In addition, a set of DOF indices to be used as the master DOF is defined as active DOF, designated as $\mathcal{A}$, and $\mathcal{A}=\mathcal{B} \cup \mathcal{O}$. Now consider an eigenvalue problem of the system Eq. (2), where the eigenvalue $\lambda$ and the corresponding eigenvector $\phi$ must satisfy the following:

$$
\left(\begin{array}{ll}
\mathbf{K}_{a a} & \mathbf{K}_{a d} \\
\mathbf{K}_{d a} & \mathbf{K}_{d d}
\end{array}\right)\left[\begin{array}{c}
\phi_{a} \\
\boldsymbol{\phi}_{d}
\end{array}\right]=\lambda\left(\begin{array}{ll}
\mathbf{M}_{a a} & \mathbf{M}_{a d} \\
\mathbf{M}_{d a} & \mathbf{M}_{d d}
\end{array}\right)\left[\begin{array}{c}
\phi_{a} \\
\boldsymbol{\phi}_{d}
\end{array}\right]
$$

where $\phi=\left[\phi_{a}^{\mathrm{T}}, \phi_{d}^{\mathrm{T}}\right]^{\mathrm{T}}$. In this study, a mixed-boundary CMS of Hintz-Herting ${ }^{36,37}$ is chosen for the primary model reduction. Namely, without the presence of rigid body modes, the coordinate transformation is defined as

$$
\left[\begin{array}{l}
\mathbf{x}_{a} \\
\mathbf{x}_{d}
\end{array}\right]=\mathbf{H} \boldsymbol{\eta}=\left(\begin{array}{ll}
\boldsymbol{\Psi} & \hat{\boldsymbol{\Phi}}
\end{array}\right)\left[\begin{array}{l}
\boldsymbol{\eta}_{a} \\
\boldsymbol{\eta}_{m}
\end{array}\right]
$$

where $\mathbf{x}_{a}=\boldsymbol{\eta}_{a}, \boldsymbol{\eta}_{m}$ is a vector of modal coordinates, $\boldsymbol{\Psi}$ and $\hat{\boldsymbol{\Phi}}$ are so-called constraint modes and truncated free-interface normal modes in a modified form, which are respectively defined as

$$
\begin{aligned}
& \boldsymbol{\Psi}=\left(\begin{array}{c}
\mathbf{I} \\
-\mathbf{K}_{d d}^{-1} \mathbf{K}_{d a}
\end{array}\right) \\
& \hat{\boldsymbol{\Phi}}=\left(\begin{array}{c}
\mathbf{0} \\
\mathbf{\Phi}_{d}+\mathbf{K}_{d d}^{-1} \mathbf{K}_{d a} \boldsymbol{\Phi}_{a}
\end{array}\right)
\end{aligned}
$$

and $\boldsymbol{\Phi}=\left[\phi_{(1)}, \phi_{(2)}, \ldots, \phi_{(k)}\right], k<n$, each subscript in parentheses denoting the corresponding mode number. Using the transformation defined as Eq. (4), the projected eigenvalue problem is obtained as

$$
\mathbf{K}_{H} \boldsymbol{\eta}=\mu \mathbf{M}_{H} \boldsymbol{\eta}
$$

where $\mathbf{M}_{H}=\mathbf{H}^{\mathrm{T}} \mathbf{M H}$ and $\mathbf{K}_{H}=\mathbf{H}^{\mathrm{T}} \mathbf{K H}$. It should be noted that the projected eigenvalue problem Eq. (7) possesses at least the same eigenvalues of the original systems, i.e., $\lambda_{(1)}, \lambda_{(2)}, \ldots \lambda_{(k)}$ (the indices may be different from the ones for the projected system.) This is because the subspace spanned by the columns of $(\boldsymbol{\Psi}, \hat{\boldsymbol{\Phi}})$ contains the eigenvectors of Eq. (3), i.e., $\phi_{(j)} \in \operatorname{span}(\boldsymbol{\Psi}, \hat{\mathbf{\Phi}})$ for $j=1, \ldots k$, as $\operatorname{span}(\boldsymbol{\Psi}, \hat{\boldsymbol{\Phi}})=\operatorname{span}(\boldsymbol{\Psi}, \boldsymbol{\Phi})$, and hence the projected eigenvalue problem has the same eigenvalues as the original ones. It means that, the eigenvalues of the projected system Eq. (7) does not contain any error in the eigenvalues and eigenvectors, with respect to those of the original eigenvalue problem of the finite element. Although this advantage comes with the expense of calculating the eigenvalues and eigenvectors of the finite element model, it is not a major drawback considering that the computational cost involved in the nonlinear computations with the original finite element would be more prohibitively expensive, than calculating a few normal modes of the finite element model.

\section{III.B. Nonlinear DOF sampling}

With the reduced order model obtained in III.A, the next step is to select the DOF in $\mathcal{B}$ such that the nonlinear characteristics of the system can be well approximated by applying the nonlinear boundary conditions only on the selected DOF. 


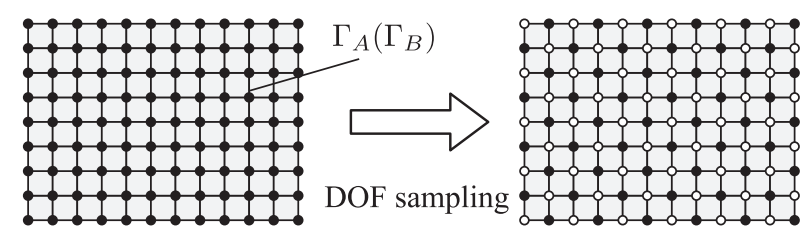

Figure 2. Schematic of the node sampling: $\bullet$, selected node $(\mathcal{N})$

As mentioned, accurately calculating the NNM frequencies is of primary interest of this study. The NNM frequencies of the system can be obtained in several ways, such as time integration of Eq. (2) for harmonic loading, or harmonic-balance-based frequency/time domain analysis. ${ }^{35,38}$ It was shown by the authors that the NNM frequencies for cracked plates obtained by the nonlinear harmonic response analysis can be well approximated by the application of bilinear frequency approximation even when the crack surfaces involve multiple DOF. ${ }^{39}$ Therefore, as a measure to evaluate the results obtained with the selected DOF, bilinear frequency is used in the following development. Namely, the $i$ th NNM frequency $\omega_{n i}$ can be approximated by a bilinear frequency $\omega_{b i}$ defined as

$$
\omega_{b i}=\frac{2 \omega_{o} \omega_{s}}{\omega_{o}+\omega_{s}}
$$

where $\omega_{o}$ and $\omega_{s}$ are the natural frequencies of the corresponding linear systems, which can be respectively obtained by solving the following eigenvalue problems:

$$
\begin{array}{ll}
\mathbf{K}_{H} \boldsymbol{\eta}=\left(\omega_{o}^{2}\right) \mathbf{M}_{H} \boldsymbol{\eta}, & \text { subject to open B.C.'s } \\
\mathbf{K}_{H} \boldsymbol{\eta}=\left(\omega_{s}^{2}\right) \mathbf{M}_{H} \boldsymbol{\eta}, & \text { subject to sliding B.C.'s }
\end{array}
$$

The open B.C. is a boundary condition where no constraint is imposed on the nodes on $\Gamma_{A}$ and $\Gamma_{B}$, or the DOF in $\mathcal{B}$. Thus in fact Eq. (9) is identical to Eq. (7). On the other hand for the sliding B.C., it is assumed that $\Gamma_{A}$ can freely slide with respect to $\Gamma_{B}$ but cannot separate along the normal directions, as described as follows.

Here a contact pair is defined as a pair of nodes on $\Gamma_{A}$ and $\Gamma_{B}$, which may or may not be in contact during the vibration, and a set of numbers denoting all the contact pairs is defined as $\mathcal{C}_{c p}$. For the $j$ th contact pair in $\mathcal{C}_{c p}$, three mutually perpendicular normal vectors at a node on $\Gamma_{A}$ are defined as $\mathbf{n}_{1}^{j}, \mathbf{n}_{2}^{j}$, and $\mathbf{n}_{3}^{j}$ where $\mathbf{n}_{1}^{j}$ is the normal vector pointing outward from the surface, $\mathbf{n}_{2}^{j}$ and $\mathbf{n}_{3}^{j}$ are unit vectors that are tangent to the surface and perpendicular to each other. Using these vectors, a coordinate transformation matrix $\mathbf{P}_{A}^{j}=\left(\mathbf{n}_{1}^{j}, \mathbf{n}_{2}^{j}, \mathbf{n}_{3}^{j}\right)$ is defined for each contact pair, with the assumption that a nodal displacement vector contains only translational DOF, such that the $x_{1}$ component of the displacement vector of the node is aligned with $\mathbf{n}_{1}^{i}$, and pointing outward from the surface. For the other node of the $j$ th contact pair on $\Gamma_{B}$, the corresponding coordinate transformation matrix that aligns the $x_{1}$ component of the nodal displacement vector with the normal vector is defined as $\mathbf{P}_{B}^{j}=-\mathbf{P}_{A}^{j}$. Now assembling $\mathbf{P}_{A}^{j}$ and $\mathbf{P}_{B}^{j}$ for all $j \in \mathcal{C}_{c p}$, a coordinate transformation is defined as

$$
\mathbf{P}=\left(\begin{array}{ccc}
\mathbf{P}_{b} & \mathbf{0} & \mathbf{0} \\
\mathbf{0} & \mathbf{I}_{o} & \mathbf{0} \\
\mathbf{0} & \mathbf{0} & \mathbf{I}_{m}
\end{array}\right), \quad \text { where } \quad \mathbf{P}_{b}=\underset{j=1}{n_{\mathcal{C}_{c p}}}\left(\mathbf{P}_{A}^{j}, \mathbf{P}_{B}^{j}\right)
$$

and $\mathrm{A}$ is an assembly operator, $\mathbf{P}_{b} \in \mathbb{R}^{n_{\mathcal{B}} \times n_{\mathcal{B}}}, \mathbf{I}_{o} \in \mathbb{R}^{n_{\mathcal{O}} \times n_{\mathcal{O}}}$, and $\mathbf{I}_{m} \in \mathbb{R}^{n_{\mathcal{M}} \times n_{\mathcal{M}}}$. Next, for the $j$ th contact pair, the $x_{1}$ components of the nodal displacement vectors, which are denoted as $\eta_{A}^{j}$ and $\eta_{B}^{j}$, are transformed 
to a relative displacement $u^{j} \triangleq\left(\eta_{A}^{j}+\eta_{B}^{j}\right) / \sqrt{2}$ and a displacement $v^{j} \triangleq\left(\eta_{A}^{j}-\eta_{B}^{j}\right) / \sqrt{2}$. Namely, denoting the set of DOF corresponding to $u_{A}^{j}$ and $u_{B}^{j}$ for all $j$ th contact pair,

$$
\left[\begin{array}{l}
u^{j} \\
v^{j}
\end{array}\right]=\frac{1}{\sqrt{2}}\left(\begin{array}{cc}
1 & 1 \\
1 & -1
\end{array}\right)\left[\begin{array}{l}
\eta_{A}^{j} \\
\eta_{B}^{j}
\end{array}\right]
$$

Now defining sets $\mathcal{X}, \mathcal{Y}$, and $\mathcal{Z}\left(\mathcal{X} \cup \mathcal{Y} \cup \mathcal{Z}=\mathcal{B}\right.$ and $\left.n_{\mathcal{X}}=n_{\mathcal{Y}}=n_{\mathcal{Z}}=n_{\mathcal{C}_{c p}}\right)$ that respectively contain sets of indices of the DOF corresponding to $x_{1}, x_{2}$, and $x_{3}$ for all $j \in \mathcal{C}_{c p}$, and denoting the coefficient matrix in the Eq. (12) as $\mathbf{R}^{j}$, one can define a transformation matrix $\mathbf{R}$ by assembling $\mathbf{R}^{j}$ for $j \in \mathcal{C}_{c p}$ as follows

$$
\mathbf{R}=\left(\begin{array}{cc}
\mathbf{R}_{x} & \mathbf{0} \\
\mathbf{0} & \mathbf{I}
\end{array}\right), \quad \text { where } \quad \mathbf{R}_{x}=\mathrm{A}_{j=1}^{n_{\mathcal{C}_{c p}}}\left(\mathbf{R}^{j}\right)
$$

and $\mathbf{R}_{x} \in \mathbb{R}^{n_{\mathcal{X}} \times n_{\mathcal{X}}}$. Considering that $\mathbf{P}^{-1}=\mathbf{P}^{\mathrm{T}}$ and $\mathbf{R}^{-1}=\mathbf{R}^{\mathrm{T}}$, the eigenvalue problem Eq. (7) can be transformed to

$$
\mathbb{K} \mathbf{q}=\left(\omega_{o}^{2}\right) \mathbb{M} \mathbf{q}
$$

where $\boldsymbol{\eta}=\mathbf{P R q}, \mathbb{M}=(\mathbf{P R})^{\mathrm{T}} \mathbf{M}_{H} \mathbf{P R}$, and $\mathbb{K}=(\mathbf{P R})^{\mathrm{T}} \mathbf{K}_{H} \mathbf{P R}$. Now noting that $\mathbf{q}$ can be partitioned into $\mathbf{q}=\left[\mathbf{q}_{r}, \mathbf{q}_{g}\right]$ where $\mathbf{q}_{r}$ is the vector of relative DOF, or $u^{j}, \forall j \in \mathcal{C}_{c p}$, and $\mathbf{q}_{g}$ is the generalized internal DOF containing $v^{j}, \forall j \in \mathcal{C}_{c p}, x_{2}$ and $x_{3}$ components of the nodal displacement vectors of the nodes of the contact pairs, displacement vectors of the observer nodes, and modal coordinates. That is, Eq. (14) can be written as

$$
\left(\begin{array}{ll}
\mathbb{K}_{r r} & \mathbb{K}_{r g} \\
\mathbb{K}_{g r} & \mathbb{K}_{g g}
\end{array}\right)\left[\begin{array}{l}
\mathbf{q}_{r} \\
\mathbf{q}_{g}
\end{array}\right]=\left(\omega_{o}^{2}\right)\left(\begin{array}{ll}
\mathbb{M}_{r r} & \mathbb{M}_{r g} \\
\mathbb{M}_{g r} & \mathbb{M}_{g g}
\end{array}\right)\left[\begin{array}{l}
\mathbf{q}_{r} \\
\mathbf{q}_{g}
\end{array}\right]
$$

where $\mathcal{R} \subset \mathcal{X}$ and $\mathcal{G}=(\mathcal{A} \backslash \mathcal{R}) \cup \mathcal{M}$.

The best approximation to the NNM frequency can be obtained when the sliding boundary conditions are imposed on all of the nodes on the surface $\Gamma_{A}$ and $\Gamma_{B}$. Namely the associated eigenvalue problem with the sliding boundary conditions can be obtained by constraining all the relative DOF, or $\mathbf{q}_{r}=\mathbf{0}$, i.e.,

$$
\mathbb{K}_{g g} \mathbf{q}_{g}=\left(\omega_{s}^{2}\right) \mathbb{M}_{g g} \mathbf{q}_{g}
$$

Now, we assume that we do not like to consider all nodes in $\mathcal{R}$ for the subsequent forced response analysis due to the large number of DOF involved in $\mathcal{R}$. In other words, the nodes where the nonlinear boundary conditions are applied should be sampled such as illustrated in Fig. 2. The selected DOF is designated as nonlinear DOF, and a set of indices of the nonlinear DOF is denoted as $\mathcal{N}$, where $\mathcal{N} \subset \mathcal{R}$. The rest of DOF in $\mathcal{R}$ is designated as linear DOF, and associated set is denoted as $\mathcal{L}$ where $\mathcal{N} \cup \mathcal{L}=\mathcal{R}$. Therefore, the bilinear frequency should be calculated with $\omega_{s}$ such that the sliding B.C. is applied only on the DOF in $\mathcal{N}$, or $\mathbf{q}_{n}=\mathbf{0}$, i.e.,

$$
\left(\begin{array}{ll}
\mathbb{K}_{l l} & \mathbb{K}_{l g} \\
\mathbb{K}_{g l} & \mathbb{K}_{g g}
\end{array}\right)\left[\begin{array}{l}
\mathbf{q}_{l} \\
\mathbf{q}_{g}
\end{array}\right]=\left(\omega_{s}^{2}\right)\left(\begin{array}{ll}
\mathbb{M}_{l l} & \mathbb{M}_{l g} \\
\mathbb{M}_{g l} & \mathbb{M}_{g g}
\end{array}\right)\left[\begin{array}{l}
\mathbf{q}_{l} \\
\mathbf{q}_{g}
\end{array}\right]
$$

Considering that the value of the natural frequency of the system with the open boundary conditions, $\omega_{o}$, is independent on neither the number nor the pattern of the selected DOF (recalling that $\operatorname{span}\left(\boldsymbol{\Psi}_{c}, \hat{\Phi}\right)$ contains the chosen eigenvectors), one can see from Eq. (8) that $\omega_{b i}$ is dependent only on $\omega_{s}$ for a fixed $\omega_{o}$. Now considering the Rayleigh's theorem of constraints defined by Eq. (1), it is known that all the system's eigenvalues increase if a single constraint is imposed on a system. Therefore, as the number of constraints on Eq. (7) to calculate $\omega_{s}$ increases, $\omega_{s}$ increases. Furthermore, considering that $\omega_{b i}$ is a monotonically increasing function of $\omega_{s}$ for a fixed $\omega_{o}$, or $\partial \omega_{b i} / \partial \omega_{s}=2 \omega_{o}^{2} /\left(\omega_{o}+\omega_{s}\right)^{2} \geqslant 0$, one can state that the best 
approximation of $\omega_{b i}$ for a given number of $n_{\mathcal{N}}$ can be obtained when the maximum $\omega_{s}$ is achieved. Thus a corresponding maximization problem is stated as follows:

$$
\begin{gathered}
\max _{\mathcal{N} \subset \mathcal{R}} \omega_{s}(\mathcal{N}) \\
\text { subject to }|\mathcal{N}|=n_{\mathcal{N}}
\end{gathered}
$$

This maximization problem may be solved by mathematical programming methods, such as integer programming or topology optimization methods as was done in Ref. ${ }^{27}$ As it shall be discussed next, this maximization problem can in fact be treated in a more efficient way by the use of Guyan reduction and some methods to choose the master DOF for reduced order modeling techniques.

\section{III.C. Automatic master DOF selection}

The methods for automatically selecting the master DOF for the Guyan reduction have been previously developed, such as in Refs. ${ }^{9}, 10,16$ In particular, the method proposed by Henshell and Ong ${ }^{9}$ appears to be the most successful approach. Although it has been known to be computationally expensive due to the nature of eliminating a single DOF per iteration and successive application of Guyan reduction, this can be alleviated by the application of the primary model reduction by the CMS as developed in III.A. As was mentioned by Bouhaddi and Fillod, ${ }^{16}$ and Shah and Raymund, ${ }^{10}$ the master DOF of Guyan reduction should be chosen such that the valid eigenvalue range of the reduced order model is maximized. In general, it has been known that the eigenvalue range of validity is "bounded" by the lowest eigenvalue of the system with all the master DOF fixed. Here this concept is applied to the problem of finding the optimal $\mathcal{N}$ that solves Eq. (18). Namely, the corresponding eigenvalue problem is Eq. (17) by regarding $\mathbf{q}_{n}$ as the master DOF. As was discussed in the Ref., ${ }^{40}$ the error bounds in the $i$ th eigenvalue of the reduced model produced by the Guyan reduction can be obtained a priori by the following relationship

$$
0 \leqslant \varepsilon_{i} \leqslant \frac{\lambda_{i}}{\lambda_{s, \min }-\lambda_{i}}
$$

where $\varepsilon_{i} \triangleq\left(\bar{\lambda}_{i}-\lambda_{i}\right) / \lambda_{i}$ is the relative error in the $i$ th eigenvalue, $\bar{\lambda}_{i}$ is the $i$ th eigenvalue of the reduced order model, $\lambda_{i}$ is the $i$ th eigenvalue of the original finite element model, and $\lambda_{s, \text { min }}$ is the smallest eigenvalue of the system with all the master DOF fixed. For $\lambda_{i} / \lambda_{s, \min } \ll 1$, the upper bound asymptotically converges to the following value, ${ }^{41}$

$$
0 \leqslant \varepsilon_{i} \leqslant \lambda_{i} / \lambda_{s, \min }
$$

Therefore, it is apparent that maximizing $\lambda_{s, \min }$ results in minimizing the upper bound of the error for all the eigenvalues of the reduced order model. Hence this gives us a guideline for selecting the master DOF for Guyan reduction such that the errors in the eigenvalues of the resulting reduced order model are minimized.

By observing this fact from another point of view, one may see that if a certain set of master DOF can achieve the maximum $\lambda_{s, \min }$, we can obtain not only an accurate reduced order model that can well approximate the first few lowest eigenvalues of the original system, but also as a "byproduct", a good estimate on the optimal constraint locations that maximize the fundamental frequency. Recasting this to our original problem of selecting the optimal set $\mathcal{N}$, the error bounds Eq. (20) associated with the eigenvalue problem Eq. (17) are written as

$$
0 \leqslant \varepsilon_{i} \leqslant \frac{\left(\omega_{o}^{2}\right)_{i}}{\left(\omega_{s}^{2}\right)_{1}-\left(\omega_{o}^{2}\right)_{i}}
$$

where $\varepsilon_{i} \triangleq\left[\left(\bar{\omega}_{o}^{2}\right)_{i}-\left(\omega_{o}^{2}\right)_{i}\right] /\left(\omega_{o}^{2}\right)_{i},\left(\bar{\omega}_{o}^{2}\right)_{i}$ is the $i$ th eigenvalue of a reduced order model, $\left(\omega_{s}\right)_{1}$ is the lowest natural frequency of Eq. (17). The corresponding maximization problem is Eq. (18), and by solving this problem for the lowest eigenvalue, $\left(\omega_{s}\right)_{1}$, one can expect that the chosen nodes pattern is at least suboptimal. 


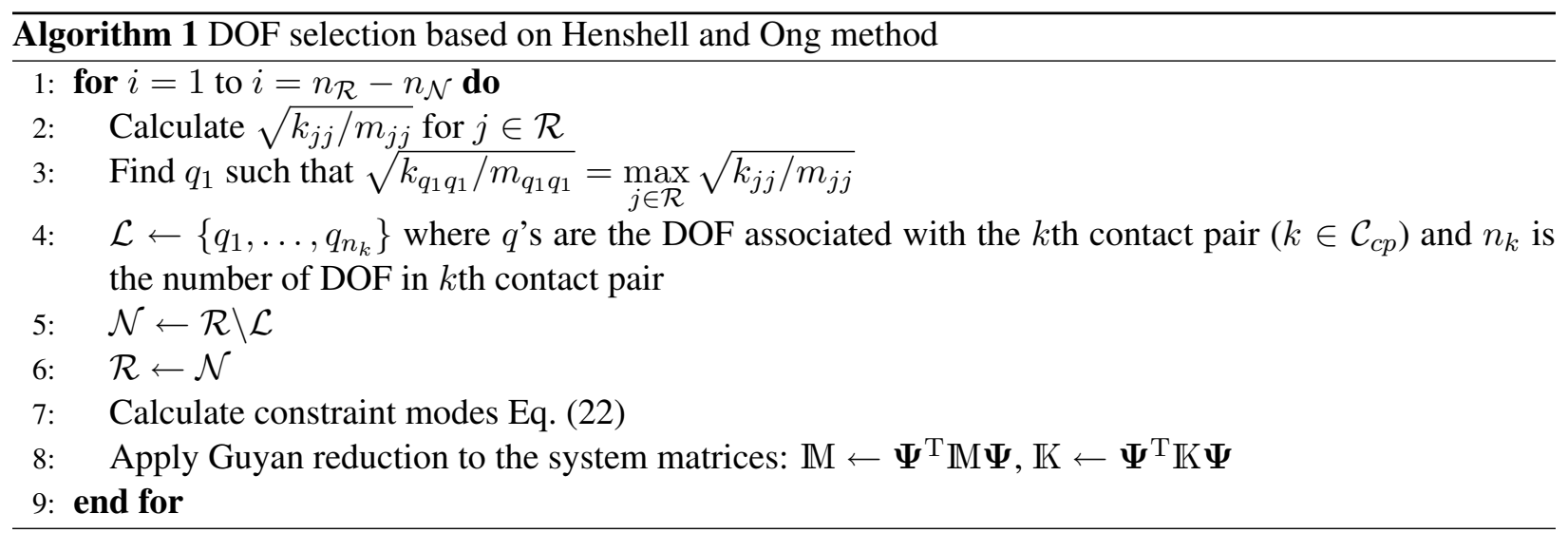

According to Refs., ${ }^{41,42}$ the sequential elimination method by Henshell and Ong ${ }^{9}$ tends to keep $\lambda_{s, \min }$ high, as it eliminates the DOF associated with the highest constrained frequency at each iteration as the slave DOF. Namely after the elimination procedure, if the chosen master DOF are all fixed, the system is left with the DOF that were chosen as the slave DOF that were identified to have the highest constrained frequency at each elimination process. Thus the resulting system with all the master DOF fixed tends to have a larger $\lambda_{s, \min }$ than that calculated with systems with other possible combinations of master DOF fixed. The Henshell and Ong's method that is adapted specifically for this problem is shown in Algorithm 1. First, at each iteration, the ratios of the diagonal terms of the stiffness matrix $k_{j j}$ to the diagonal terms of the mass matrix $m_{j j}$ are calculated for $\forall j \in \mathcal{R}$, and the index $q_{1}$ that gives the maximum ratio among $j \in \mathcal{R}$ is obtained. Next, the set $\mathcal{L}$ is updated such that it contains $q_{1}$, and all the other DOF that are associated with the contact pair $k \in \mathcal{C}_{c p}$ to which the $q_{1}$ th DOF belongs, e.g., the DOF that are perpendicular to the normal direction. The set $\mathcal{N}$ is then updated such that it excludes the selected DOF of $\mathcal{L}$ from $\mathcal{R}$, and the set $\mathcal{R}$ is re-defined as $\mathcal{N}$. A constraint mode is calculated by solving a problem where a unit displacement is applied to a DOF in $\mathcal{N}$ whereas all the other DOF in $\mathcal{N}$ being fixed. This is repeated for all DOF in $\mathcal{N}$, resulting in the following matrix:

$$
\boldsymbol{\Psi}=\left(\begin{array}{c}
\mathbf{I} \\
-\left(\mathbb{K}_{l l}\right)^{-1} \mathbb{K}_{l n}
\end{array}\right)
$$

where $\Psi$ is the matrix of constraint modes for all DOF in $\mathcal{N}$. The Guyan reduction is then applied to the mass and stiffness matrices. The iteration continues until the number of DOF in $\mathcal{N}$ reaches the specified value of $n_{\mathcal{N}}$ using $\Psi$.

In order to clarify the appropriateness of the algorithm in Algorithm 1 to this problem, another algorithm for selecting DOF is shown here for comparison. The method of effective independence vector, or the EIDV method developed by Kammer, ${ }^{26}$ is a method to choose the sensor placement locations for the vibration measurement of large scale structures. The method aims to make the measured eigenvectors as linearly independent as possible. According to Penny et al. ${ }^{25}$ many of the criteria for choosing the master DOF for model order reduction have similar criteria for choosing measurement locations in a way such that the lower frequency modes can be captured accurately. In fact, as examined by Penny et al., both the Henshell and Ong method and the EIDV method produce acceptable selections in most cases, in a suboptimal manner. The DOF selection algorithm based on the EIDV method is shown as Algorithm 2. First, the eigenvalue problem Eq. (16) is solved for the first $k$ modes, and the associated modal matrix is designated as $\boldsymbol{\Phi}_{k}=\left(\phi_{1}, \phi_{2}, \ldots, \phi_{k}\right)$, or $\mathbb{K} \boldsymbol{\Phi}_{k}=\mathbb{M} \boldsymbol{\Phi}_{k} \boldsymbol{\Lambda}_{k}$ where $\boldsymbol{\Lambda}_{k}=\operatorname{diag}_{j=1, \ldots, k}\left(\left(\omega_{o}^{2}\right)_{j}\right)$. The Fisher information matrix $\mathbf{A}$ is then calculated as $\mathbf{A}=\boldsymbol{\Phi}_{k}^{\mathrm{T}} \boldsymbol{\Phi}_{k}$, and an idempotent matrix $\mathbf{E}$ is computed as $\mathbf{E}=\boldsymbol{\Phi}_{k} \mathbf{A}^{-1} \boldsymbol{\Phi}_{k}^{\mathrm{T}}$, the diagonal of which is called the independence distribution vector (see Ref. ${ }^{26}$ for detailed formulations.) The least contributing DOF to the independence of the modes among the ones in $\mathcal{R}$ is identified as the one with the smallest diagonal element in $\mathbf{E}$. The associated DOF are also identified and stored in $\mathcal{L}$, and both 


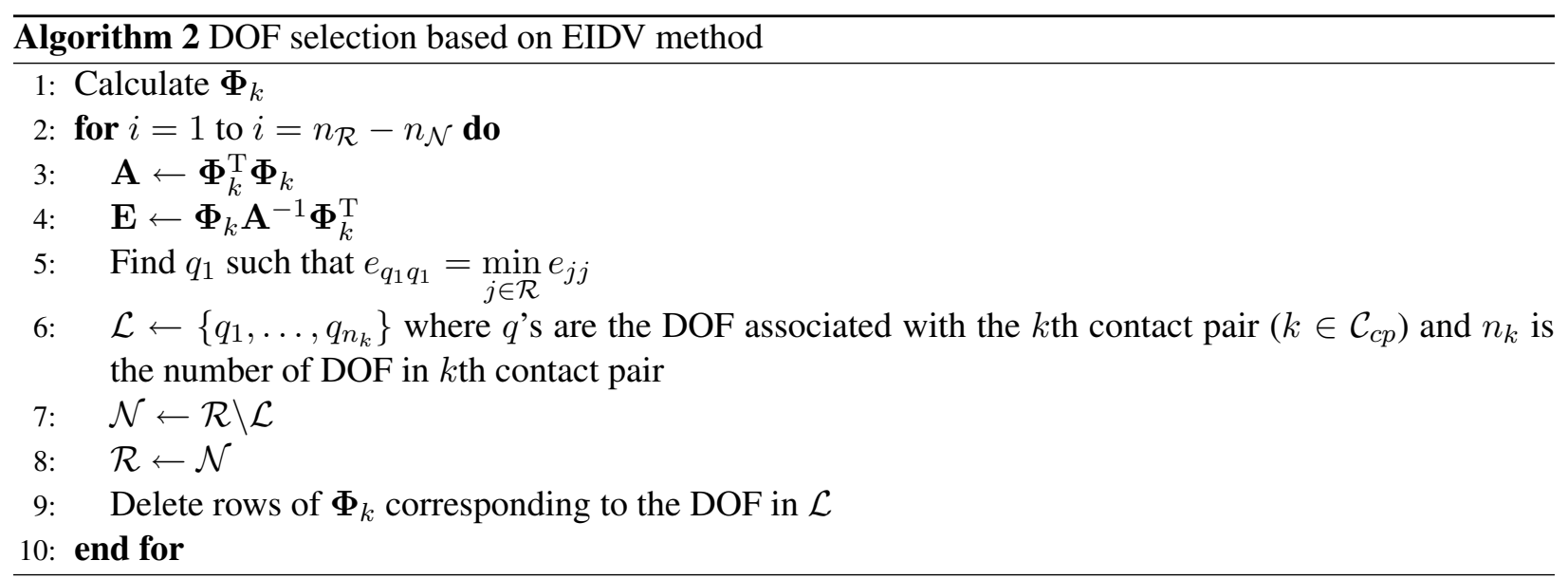

$\mathcal{N}$ and $\mathcal{R}$ are updated as in the Henshell and Ong method. Finally the rows of $\boldsymbol{\Psi}_{k}$ corresponding to the DOF in $\mathcal{L}$ are deleted. The iteration continues until the size of $\mathcal{N}$ reaches the desired number $n_{\mathcal{N}}$.

Although the EIDV method shares similar objective for choosing DOF with the Henshell and Ong method, the objective of the EIDV method is not exactly the maximization problem of Eq. (18). Therefore it is expected that the Henshell and Ong method returns better solutions to the given maximization problem than the EIDV method, as it is shown in the next section.

\section{Case study}

In section III, the method to select the nonlinear DOF has been introduced. In this section, the validity and applicability of the method are discussed by applying the algorithm to an example problem. With the case study, the validity of the proposed method is discussed in terms of the bilinear frequencies and forced response. Furthermore a metric to assess their accuracy is introduced and examined.

\section{IV.A. Simple cracked plate model}

\section{IV.A.1. Problem description}

A cantilevered cracked plate model was constructed with Young's modulus $E=2.0 \times 10^{11} \mathrm{~Pa}$, Poisson's ratio $\nu=0.3$, and density $\rho=7800 \mathrm{~kg} / \mathrm{m}^{3}$, and its geometry is shown as Fig. 3a where $w=6.0 \times 10^{-3} \mathrm{~m}$, $l=6.0 \times 10^{-2} \mathrm{~m}, h=1.5 \times 10^{-1} \mathrm{~m}, l_{c} / l=0.625$, and $h_{c} / h=0.475$. The model was discretized with 5,120 linear solid elements and resulted in mass and stiffness matrices with 18,630 DOF. On the crack surfaces as shown in Fig. 3b, there are 180 nodes, or 90 contact pairs on the surfaces hence the number of the associated DOF is 540. The CMS method shown in the section III.A was then applied to the FE model, and it resulted in the $681 \mathrm{DOF}$ (3.6\% of the original size) system consisting of 621 physical DOF and 60 modal coordinates corresponding to the free-interface normal modes. With this reduced order model, both algorithms in Figs. 1 and 2 were applied for $n_{\mathcal{N}}=4,8,16,32,64$, and 128. For the EIDV method, the first four modes were considered.

In order to compare these results with an "intuitive" selection method, a selection criteria was also employed, where the nonlinear DOF were chosen based on the amount of penetration between the nodes in a contact pair for the modes of interest, which in this case is the fourth mode. Namely, it was hoped that penalizing the inter-penetration of the most penetrating contact pairs would produce the "stiffest" system response. The selected node pattern with such criterion is shown as Fig. 4, and the results of the EIDV method and the Henshell and Ong method are shown as Figs. 5 and 6. As can be seen in Fig. 4, if the nodes are chosen based on the amount of penetration, the selection starts from the nodes near the crack edge (open 


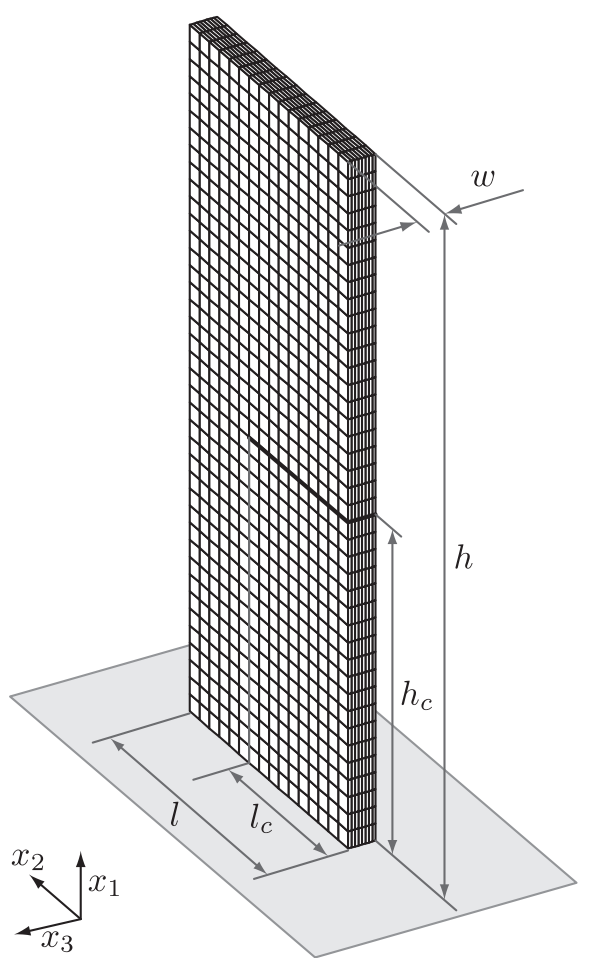

(a)

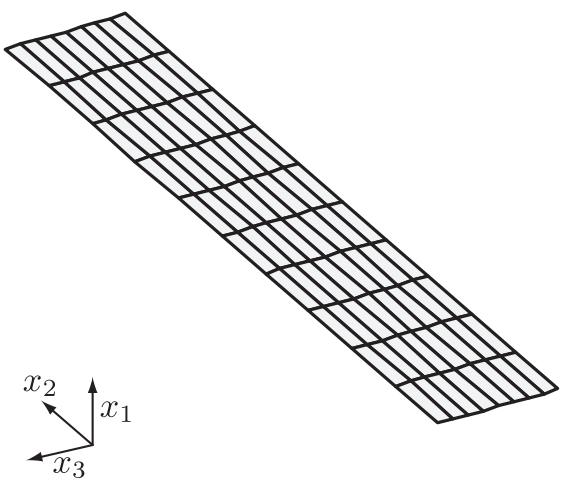

(b)

Figure 3. Cantilevered cracked plate model: (a) FE model, (b) Magnified crack surface

side) for $n_{\mathcal{N}}=4$, and it then proceeds toward the tip of the crack (closed side) as $n_{\mathcal{N}}$ increases. It makes sense because the motion of the crack surfaces is significant near the open edge than that near the closed edge. On the other hand with the EIDV method, the method also starts to select the nodes near the crack edge, but it tends to choose more nodes on the crack rims than the nodes near the crack edge as shown in Fig. 5. Finally with the Henshell and Ong method, it also select the nodes near the crack edge first, for $n_{\mathcal{N}}=4$, but it then tends to select the nodes over the crack surface in a more distributed manner as can be seen in Fig. 6.

\section{IV.A.2. Forced Response Analysis}

Next, in order to see the influence of the application of the nonlinear B.C. onto the selected nodes on an NNM frequency, forced response analysis was carried out by applying an external harmonic loading to the cracked plate. As one might notice, when the forced response of this structure with a crack is considered, the

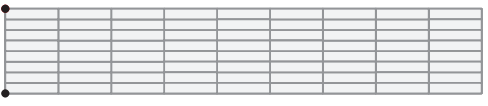

(a) $n_{\mathcal{N}}=4$ (2 pairs)

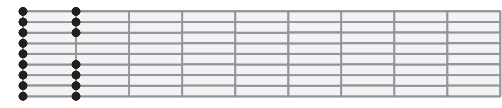

(d) $n_{\mathcal{N}}=32$ (16 pairs)

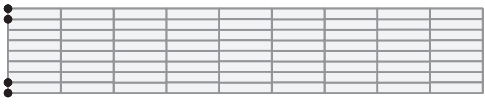

(b) $n_{\mathcal{N}}=8$ (4 pairs)

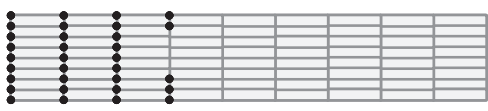

(e) $n_{\mathcal{N}}=64$ (32 pairs)

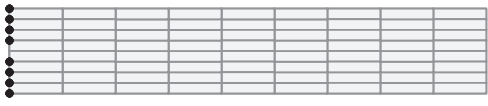

(c) $n_{\mathcal{N}}=16$ (8 pairs)

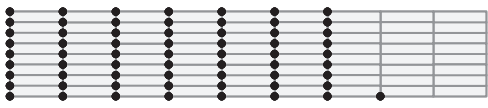

(f) $n_{\mathcal{N}}=128$ (64 pairs)

Figure 4. Selected nodes by an intuitive approach (left edge open) 


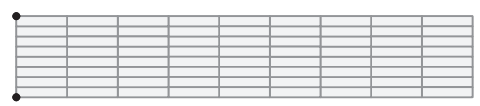

(a) $n_{\mathcal{N}}=4$ (2 pairs)

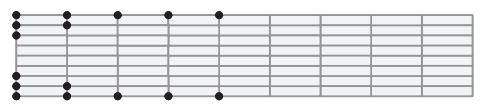

(d) $n_{\mathcal{N}}=32$ (16 pairs)

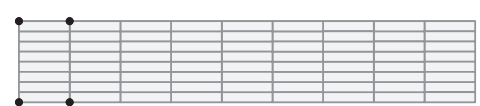

(b) $n_{\mathcal{N}}=8$ (4 pairs)

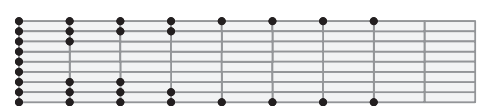

(e) $n_{\mathcal{N}}=64$ (32 pairs)

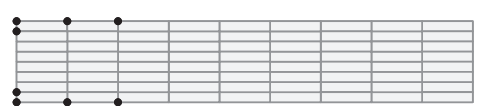

(c) $n_{\mathcal{N}}=16$ (8 pairs)

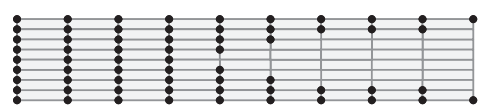

(f) $n_{\mathcal{N}}=128$ (64 pairs)

Figure 5. Selected nodes by EIDV method (left edge open)

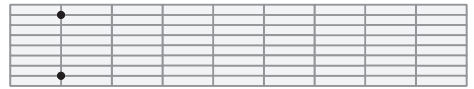

(a) $n_{\mathcal{N}}=4$ (2 pairs)

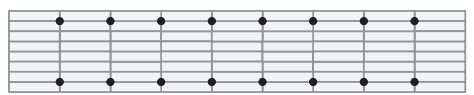

(d) $n_{\mathcal{N}}=32$ (16 pairs)

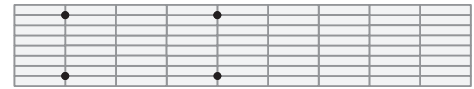

(b) $n_{\mathcal{N}}=8$ (4 pairs)

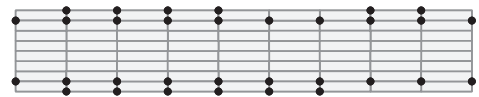

(e) $n_{\mathcal{N}}=64$ (32 pairs)

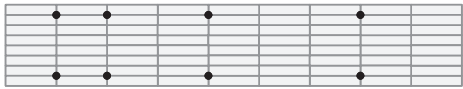

(c) $n_{\mathcal{N}}=16$ (8 pairs)

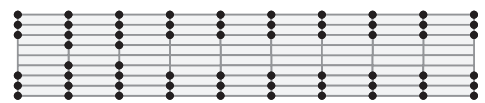

(f) $n_{\mathcal{N}}=128$ (64 pairs)

Figure 6. Selected nodes by the modified Henshell and Ong method (left edge open)

repetitive opening and closing of the crack faces must be treated appropriately with contact algorithms. As a result, the vibration is nonlinear and the steady-state response of the displacement may not be expressed as a harmonic function even if the external force is a harmonic function. Therefore in this study, the steady-state response was obtained by assuming that the displacement can be expressed as a truncated Fourier series, and the nonlinear boundary condition can be enforced by the penalty method. ${ }^{1}$ The method is called the hybrid frequency-time domain method, ${ }^{35,38}$ which is based on the concept of harmonic balance method. ${ }^{4}$ Detailed formulation of the method is omitted in this paper.

It is noted that the system matrices were further reduced by the application of Eq. (4) to the reducedorder model before the forced response calculation, by keeping the selected node pairs as the active DOF and condensing out the other DOF including physical and modal coordinates. For example, with $n_{\mathcal{N}}=64$ (32 pairs), the system size was reduced down to $155 \mathrm{DOF}$, which is $0.83 \%$ of the original system size.

A harmonic forcing of magnitude $3 \mathrm{~N}$ was then applied at the tip of the plate, in order to excite the first vibration mode, which corresponds to the first out-of-plance bending mode. The forced response was calculated for both linear case, i.e., with the open B.C., and nonlinear case with the nonlinear boundary conditions imposed on $\mathcal{N}$ with the selections by the Henshell and Ong method. The results are shown in Figs. 7a and 7b. As can be seen in Fig 7a, the selection pattern does not alter the linear forced response. This is because the selection of the active DOF does not alter the eigenvalues of the reduced order model, and it was assumed that the system was completely linear when the forced response was calculated. On the other hand, the number of contact pairs greatly affects the results of nonlinear forced response as shown in Fig. 7b. One may observe that the response with 64 contact pairs is almost identical to that with the full set of 90 contact pairs, which implies that for accurately calculating the nonlinear resonant frequencies, it may not be necessary to enforce nonlinear boundary conditions for all the contact pairs on the crack faces. The same forced response calculations were carried out with the node patterns selected by the EIDV method and the method based on the amount of penetration, and they are respectively shown in Figs. 7c and 7d. As can be seen in Fig. 7c, the results with the patterns chosen by the EIDV method are comparable with the ones produced by the Henshell and Ong method. On the other hand as can be seen in Fig. 7d, the forced response with the node patterns chosen by the "intuitive" approach produced worse results than the other two methods, i.e., for a given number of $n_{\mathcal{N}}$, the predicted resonant frequency by the approach is lower than 


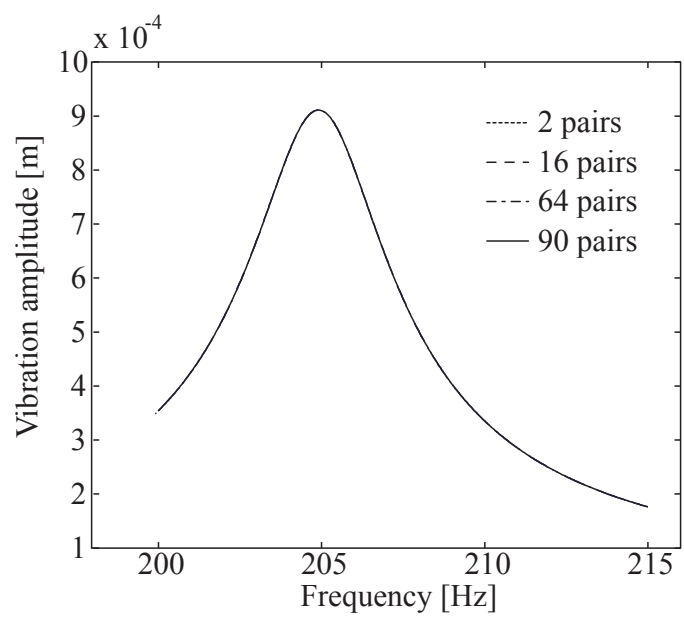

(a) Henshell and Ong method (linear)

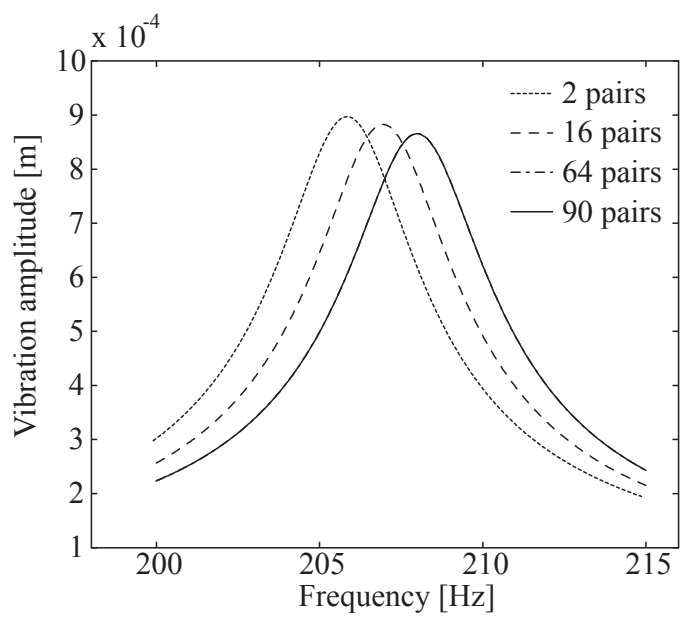

(c) EIDV method (nonlinear)

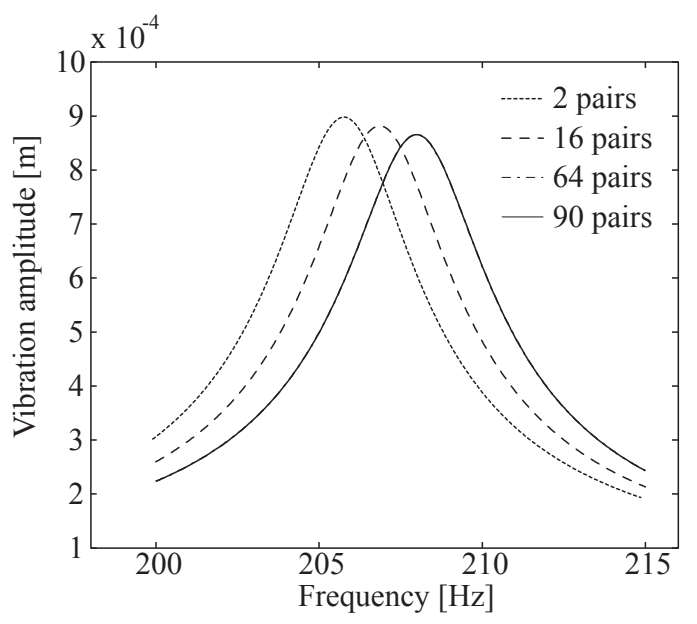

(b) Henshell and Ong method (nonlinear)

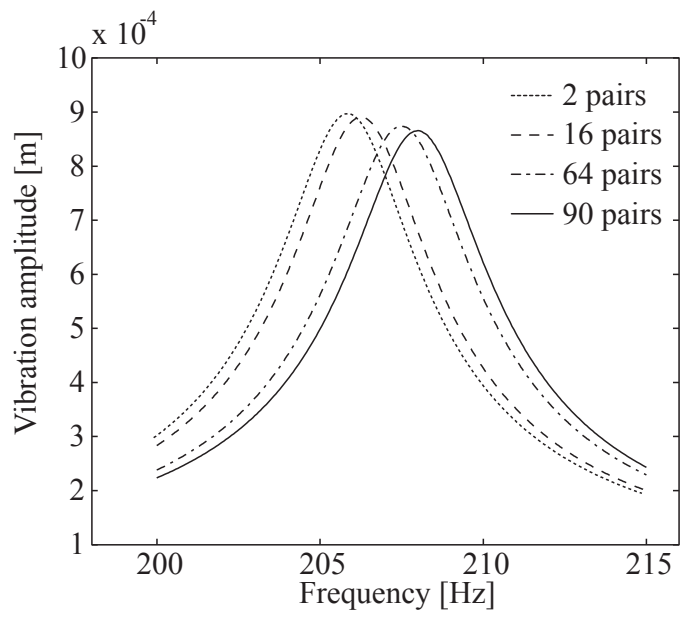

(d) Intuitive approach (nonlinear)

Figure 7. Results of forced response analysis of the cracked plate

that calculated by the other methods. This is the most visible in the results for $n_{\mathcal{N}}=64$, for which both the Henshell and Ong method and the EIDV methods produced results that are almost identical to the results for $n_{\mathcal{N}}=90$.

\section{IV.A.3. Bilinear Frequency Approximation}

Finally, the influence of the selected node pattern on the bilinear frequencies is discussed. The first four bilinear frequencies were calculated for the model with the selected node patterns with the three methods, and the results are shown in Fig. 8. The first four modes correspond to the first out-of-plane bending, the first torsion, the second out-of-plane bending, and the first in-plane bending modes respectively. The plots in Fig. 8 show the percentage errors in the bilinear frequency versus the number of contact pairs, where the error is defined as the ratio of the difference between the bilinear frequency with the sampled contact pairs and that with the full set of contact pairs, to that with the full set of contact pairs. As can be seen, the Henshell and Ong method consistently provides the best results among all the methods for the first four modes. Moreover, it shows the best convergence rate in terms of the number of contact pairs. 


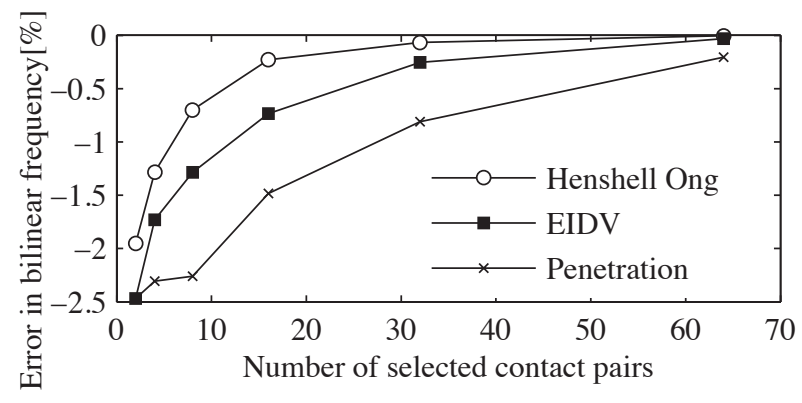

(a)

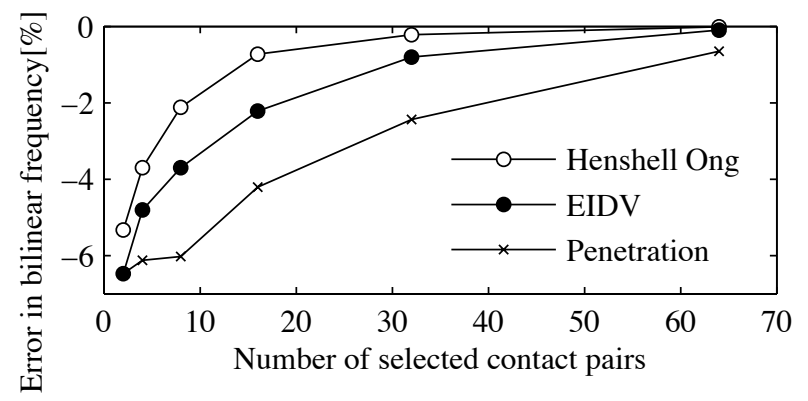

(c)

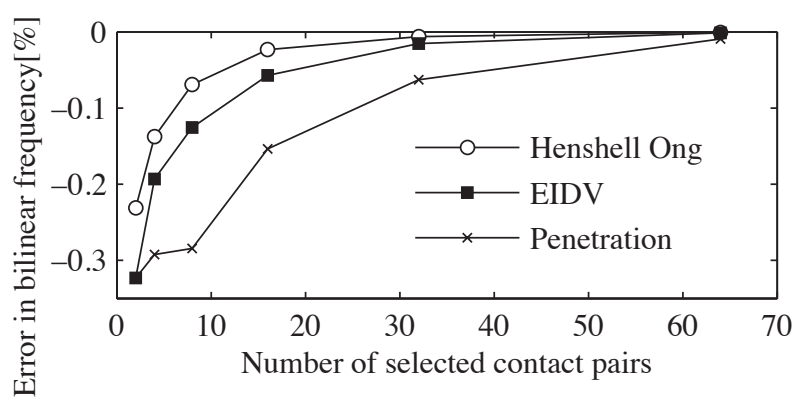

(b)

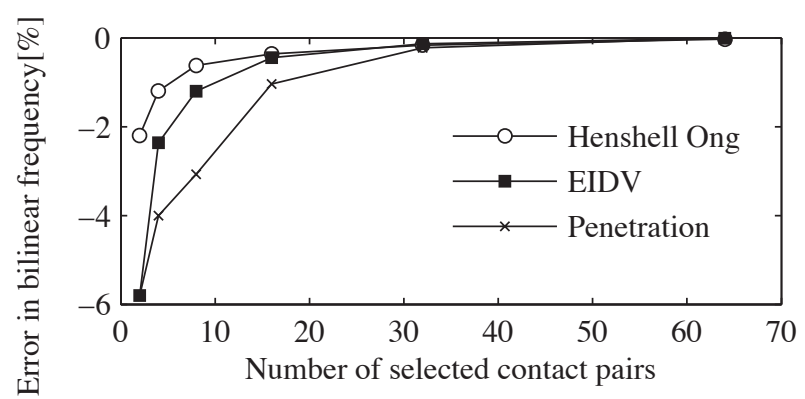

(d)

Figure 8. Errors in the first four bilinear frequencies:(a) NNM 1, (b) NNM 2, (c) NNM 3, (d) NNM 4

\section{IV.A.4. A posteriori accuracy assessment}

As seen above, even though the intuitive approach chooses the contact pairs that show the most penetration, application of the nonlinear boundary conditions to these nodes does not result in the "stiffest" vibration response. To be specific, the Henshell and Ong method and the EIDV method produced the node patterns that yield the closer results to the reference results in terms of forced response and bilinear frequencies, than the patterns chosen by the intuitive approach. In particular, the Henshell and Ong method iteratively aims to solve the maximization problem Eq. (18) in a sub-optimal manner. Therefore the bilinear frequencies as well as the resonant frequencies were well approximated with the nodes chosen by the Henshell and Ong method. In order to better understand the governing factor for the accuracy of the results, a more physical interpretation of the results is provided here. Namely, the key effect for achieving the good approximation of the NNM frequency is to ensure, as much as possible, the non-penetrability condition on the contact pairs where the nonlinear B.C.s' are not applied. The penetration should be evaluated during a vibration cycle, thus both the depth and the duration of the penetration should be taken into account. These quantities vary in space, and depend on the frequency of vibration. Hence as a metric to characterize not only the amount but also the duration of penetration over the entire crack surfaces for a given vibration frequency, the following quantity is introduced:

$$
\hat{F}=\int_{0}^{T}\left(\int_{\Gamma_{A}\left(\Gamma_{B}\right)} k_{e} u_{p}(\mathbf{r}, t) \mathrm{d} \Gamma\right) \mathrm{d} t
$$

where $\hat{F}$ is a quantity with the dimension of impulse named "virtual impulse", $k_{e}$ is an equivalent spring constant per unit length determined by the ratio between the Young's modulus multiplied by the characteristic area and the characteristic length, $u_{p}$ is the amount of penetration along the surface normals, and $T$ is the period of vibration associated with the NNM frequency. The quantity $\hat{F}$ is calculated based on the calculated time trajectory of displacements of the nodes on the crack surfaces, and can be thought of as an impulse that does not contribute to the system response, as this impulse is not applied to the system when 


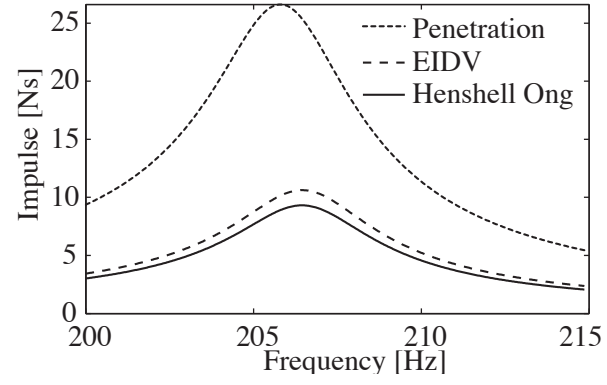

(a) $n_{\mathcal{N}}=16$ (8 pair)

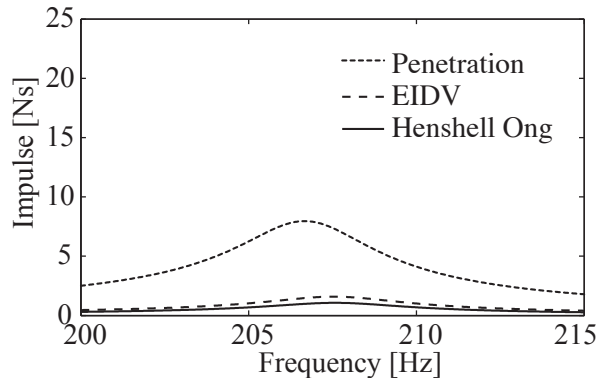

(b) $n_{\mathcal{N}}=64$ (32 pair)

Figure 9. Virtual impulse for a period of vibration for NNM 1

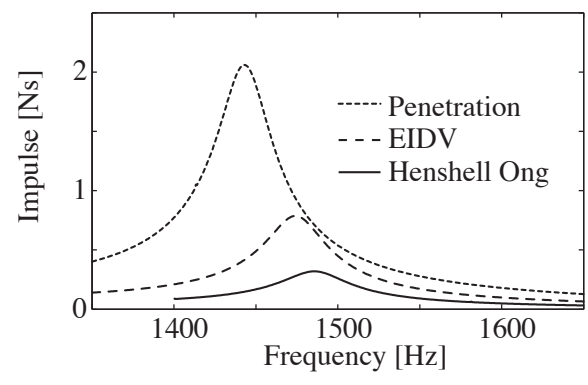

(a) $n_{\mathcal{N}}=16$ (8 pair)

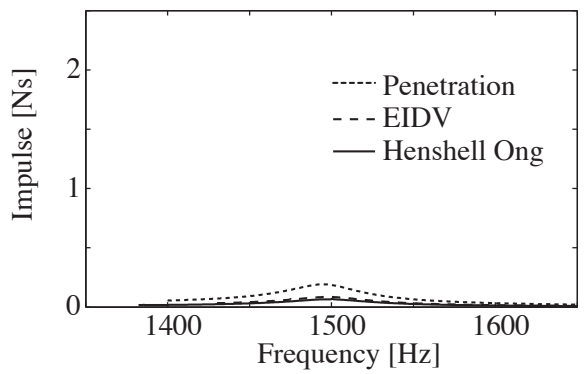

(b) $n_{\mathcal{N}}=64$ (32 pair)

Figure 10. Virtual impulse for a period of vibration for NNM 4

the response is calculated. In other words, the smaller the value of $\hat{F}$ is, the stricter the boundary conditions are imposed on the nodes over the entire crack surfaces.

First, the forced response analysis was carried out, and the corresponding time history of $u_{p}$ over the entire crack surface was recovered from the vibration response. The integrals in the Eq. (23) were then evaluated by a simple quadrature rule both in space and time. The metric was calculated for the first and the fourth modes for 8 and 32 pairs chosen by the methods, and the results are shown in Figs. 9 and 10. As can be seen in Figs. 9 and 10, the virtual impulse varies over the frequency range. In particular, when the frequency of excitation is close to the resonant frequency, or the NNM frequency, then the amount of penetration increases as well. However for all cases, the Henshell and Ong method consistently results in the smallest impulse over the frequency range among the three methods considered. It means that the nonlinear B.C. on the crack faces is the most strictly enforced by the node patterns chosen by the Henshell and Ong method.

\section{Conclusion}

In this paper, a novel reduced order modeling framework for the nonlinear vibration analysis of elastic structures with intermittent contact was proposed. In section III, the modeling framework was developed based on a method of component mode synthesis. The master DOF selection scheme for Guyan reduction was formulated by considering the close relationship between the optimal master DOF selection and the optimal constraint locations for maximizing the fundamental frequency. The method is a combination of the sequential elimination method proposed by Henshell and Ong, and appropriate coordinate transformations to the reduced order model. Another method for choosing the nodes was also introduced for the sake of comparative study, which is based on a method to optimally choose the measurement locations such 
that the measured mode becomes as linearly independent as possible. The method was then applied to a representative finite element model in section IV. In IV.A, the methods were applied to a cracked plate model. Using the selected node patterns, forced response analysis was carried out to see the effects of the selection patterns on the frequency response. Furthermore the resonant frequencies were calculated by the application of bilinear frequency approximation. It was confirmed that the selected DOF resulted in accurate prediction of nonlinear resonant frequencies in comparison to the benchmark case of using all DOF on the crack surfaces. Moreover, a posteriori accuracy assessment procedure was introduced by examining the amount of penetration on the crack surfaces during a vibration cycle. For the methods examined, the node patterns selected using the proposed new method consistently showed the best results.

\section{References}

${ }^{1}$ Oden, J. T. and Pires, E. B., "Algorithms and Numerical Results for Finite-Element Approximations of Contact Problems with Non-Classical Friction Laws," Computers \& Structures, Vol. 19, 1984, pp. 137-147.

${ }^{2}$ Simo, J. C. and Laursen, T. A., "An Augmented Lagrangian Treatment of Contact Problems Involving Friction," Computers \& Structures, Vol. 42, Jan 1992, pp. 97-116.

${ }^{3}$ Craig, R. R. and Bampton, M. C. C., "Coupling of Substructures for Dynamic Analyses," AIAA Journal, Vol. 6, No. 7, 1968, pp. 1313-1319.

${ }^{4}$ Nayfeh, A. and Mook, D., Nonlinear Oscillations, John Wiley \& Sons, 1979.

${ }^{5}$ Guyan, R. J., "Reduction of Stiffness and Mass Matrices," AIAA Journal, Vol. 3, No. 2, 1965, pp. 380.

${ }^{6}$ Brahmi, K., Bouhaddi, N., and Fillod, R., "Reduction of Junction Degrees of Freedom Before Assembly in Dynamic Substructuring," Proceedings of the ASME Design Engineering Technical Conference, Vol. 3, 1995, pp. 699-708.

${ }^{7}$ Balmés, E., "Use of Generalized Interface Degrees of Freedom in Component Mode Synthesis," Proceedings of International Modal Analysis Conference, Society for Experimental Mechanics, Bethel, CT, 1996, pp. 204-210.

${ }^{8}$ Castanier, M. P., Tan, Y. C., and Pierre, C., "Characteristic Constraint Modes for Component Mode Synthesis," AIAA Journal, Vol. 39, No. 6, 2001, pp. 1182-1187.

${ }^{9}$ Henshell, R. D. and Ong, J. H., "Automatic masters for eigenvalue economization," Earthquake Engineering \& Structural Dynamics, Vol. 3, No. 4, 1975, pp. 375-383.

${ }^{10}$ Shah, V. N. and Raymund, M., “Analytical Selection of Masters for the Reduced Eigenvalue Problem,” International Journal for Numerical Methods in Engineering, Vol. 18, No. 1, 1982, pp. 89-98.

${ }^{11}$ Kidder, R. L., "Reduction of Structural Frequency Equations," AIAA Journal, Vol. 11, 1973, pp. 892-892.

${ }^{12}$ Flax, A. H., "Comment on "Reduction of Structural Frequency Equations”," AIAA Journal, Vol. 13, 1975, pp. 701-702.

${ }^{13}$ Kidder, R. L., "Reply by Author to A. H. Flax,” AIAA Journal, Vol. 13, 1975, pp. 702-703.

${ }^{14}$ Grinenko, N. I. and Mokeev, V. V., "Problems of Studying Vibrations of Structures by the Finite-Element Method," International Applied Mechanics, Vol. 21, No. 3, 1985, pp. 231-235.

${ }^{15}$ Matta, K. W., "Selection of Degrees of Freedom for Dynamic Analysis," Journal of Pressure Vessel Technology - Transactions of the ASME, Vol. 109, 1987, pp. 65-69.

${ }^{16}$ Bouhaddi, N. and Fillod, R., "A Method for Selecting Master DOF in Dynamic Substructuring using the Guyan Condensation Method," Computers \& Structures, Vol. 45, No. 5-6, 1992, pp. 941-946.

${ }^{17}$ Hitziger, T., Mackens, W., and Voss, H., "A Condensation-Projection Method for Generalized Eigenvalue Problems," High Performance Computing 1, edited by H. Power and C. A. Brebbia, Elservier Applied Science, London, 1995, pp. $239-282$.

${ }^{18}$ Kim, K. O. and Choi, Y. J., "Energy Method for Selection of Degrees of Freedom in Condensation,” AIAA Journal, Vol. 38, 2000, pp. 1253-1259.

${ }^{19}$ Cho, M. and Kim, H., "Element-Based Node Selection Method for Reduction of Eigenvalue Problems," AIAA Journal, Vol. 42, 2004, pp. 1677-1684.

${ }^{20}$ Kim, H. and Cho, M., "Two-level Scheme for Selection of Primary Degrees of Freedom and Semi-analytic Sensitivity Based on the Reduced System," Computer Methods in Applied Mechanics and Engineering, Vol. 195, 2006, pp. 4244-4268.

${ }^{21}$ O'Callahan, J., "A procedure for an improved reduced system (IRS) model," Proceedings of the 7th International Modal Analysis Conference, Las Vegas, NV, January 1989, pp. 17-21.

${ }^{22}$ Li, W., "A Degree Selection Method of Matrix Condensations for Eigenvalue Problems," Journal of Sound and Vibration, Vol. 259, No. 2, 2003, pp. 409-425.

${ }^{23}$ Oh, D. and Park, Y., "Order Reduction Based on Singular Values of a Modal matrix," Mechanical Systems and Signal Processing, Vol. 8, No. 1, 1994, pp. 63-79. 
${ }^{24}$ Udwadia, F. E., "Methodology for Optimum Sensor Locations for Parameter Identification in Dynamic Systems," Journal of Engineering Mechanics, Vol. 120, No. 2, 1994, pp. 368-390.

${ }^{25}$ Penny, J. E. T., Friswell, M. I., and Garvey, S. D., "Automatic Choice of Measurement Locations for Dynamic Testing," AIAA Journal, Vol. 32, 1994, pp. 407-414.

${ }^{26}$ Kammer, D. C., "Sensor Placement for On-Orbit Modal Identification and Correlation of Large Space Structures," Journal of Guidance Control and Dynamics, Vol. 14, No. 2, 1991, pp. 251-259.

${ }^{27}$ Zhu, J. and Zhang, W., "Maximization of Structural Natural Frequency with Optimal Support Layout," Structural and Multidisciplinary Optimization, Vol. 31, 2006, pp. 462-469.

${ }^{28}$ ÅKesson, B. and Olhoff, N., "Minimum Stiffness of Optimally Located Supports for Maximum Value of Beam Eigenfrequencies," Journal of Sound and Vibration, Vol. 120, No. 3, 1988, pp. 457-463.

${ }^{29}$ Szelag, D. and Mroz, Z., "Optimal Design of Vibrating Beams with Unspecified Support Reactions," Computer Methods in Applied Mechanics and Engineering, Vol. 19, No. 3, 1979, pp. 333-349.

${ }^{30}$ Won, K. M. and Park, Y. S., "Optimal Support Positions for a Structure to Maximize its Fundamental Natural Frequency," Journal of Sound and Vibration, Vol. 213, No. 5, 1998, pp. 801-812.

${ }^{31}$ O'Callahan, J., Avitabile, P., and Riemer, R., "System Equivalent Reduction Expansion Process (SEREP)," Proceedings of the 7th International Modal Analysis Conference, Las Vegas, NV, January 1989, pp. 29-37.

${ }^{32}$ Friswell, M. I., Garvey, S. D., and Penny, J. E. T., "Model-Reduction using Dynamic and Iterated IRS Techniques," Journal of Sound and Vibration, Vol. 186, 1995, pp. 311-323.

${ }^{33}$ Friswell, M. I., Garvey, S. D., and Penny, J. E. T., "The Convergence of the Iterated IRS Method," Journal of Sound and Vibration, Vol. 211, 1998, pp. 123-132.

${ }^{34}$ Friswell, M. I., Penny, J. E. T., and Garvey, S. D., "Using Linear Model Reduction to Investigate the Dynamics of Structures with Local Non-Linearities," Mechanical Systems and Signal Processing, Vol. 9, No. 3, 1995, pp. 317-328.

${ }^{35}$ Saito, A., Castanier, M. P., Pierre, C., and Poudou, O., "Efficient Nonlinear Vibration Analysis of the Forced Response of Rotating Cracked Blades," Journal of Computational and Nonlinear Dynamics-Transactions of the ASME, Vol. 4, No. 1, 2009, pp. 011005 .

${ }^{36}$ Hintz, R. M., "Analytical Methods in Component Modal Synthesis," AIAA Journal, Vol. 13, No. 8, 1975, pp. $1007-1016$.

${ }^{37}$ Herting, D. N., "A General purpose, Multi-Stage, Component Modal Synthesis Method," Finite Elements in Analysis and Design, Vol. 1, No. 2, 1985, pp. 153-164.

${ }^{38}$ Poudou, O. and Pierre, C., "Hybrid Frequency-Time Domain Methods for the Analysis of Complex Structural Systems with Dry Friction Damping," Proceedings of of the 44th AIAA/ASME/ASCE/AHS/ASC Structures, Structural Dynamics, and Materials Conference, Vol. 1, Apr 2003, pp. 111-124.

${ }^{39}$ Saito, A., Castanier, M. P., and Pierre, C., "Vibration Analysis of Cracked Cantilevered Plates Near Natural Frequency Veerings," Proceedings of the 49th AIAA/ASME/ASCE/AHS/ASC Structures, Structural Dynamics, and Materials Conference, paper AIAA 2008-1872, AIAA, Reston, VA, 2008.

${ }^{40}$ Voss, H., "An Error Bound for Eigenvalue Analysis by Nodal Condensation," Numerical Treatment of Eigenvalue Problems, edited by J. Albrecht, L. Collatz, and W. Velete, Vol. 3, Birkhäuser Verlag, Stuttgart, 1983, pp. 205-214.

${ }^{41}$ Thomas, D. L., "Errors in Natural Frequency Calculations using Eigenvalue Economization," International Journal for Numerical Methods in Engineering, Vol. 18, 1982, pp. 1521-1527.

${ }^{42}$ W. Mackens, H. V., "Nonnodal Condensation of Eigenvalue Problems," Zeitschrift für Angewandte Mathematik und Mechanik (ZAMM), Vol. 79, No. 4, 1999, pp. 243-255. 